\title{
PHYSICAL PROPERTIES OF NIOBIUM AND SPECIFICATIONS FOR FABRICATION OF SUPERCONDUCTING CAVITIES
}

Prepared By:

(Claire Antoine)

APPROVED BY:

$$
(\mathrm{XXXX)}
$$




\begin{tabular}{|c|c|c|c|c|}
\hline Revision & ER / ECO & Date & Description & Approval(s) \\
\hline & & & & \\
\hline & & & & \\
\hline & & & & \\
\hline & & & & \\
\hline & & & & \\
\hline & & & & \\
\hline & & & & \\
\hline & & & & \\
\hline & & & & \\
\hline
\end{tabular}




\section{TOPICS}

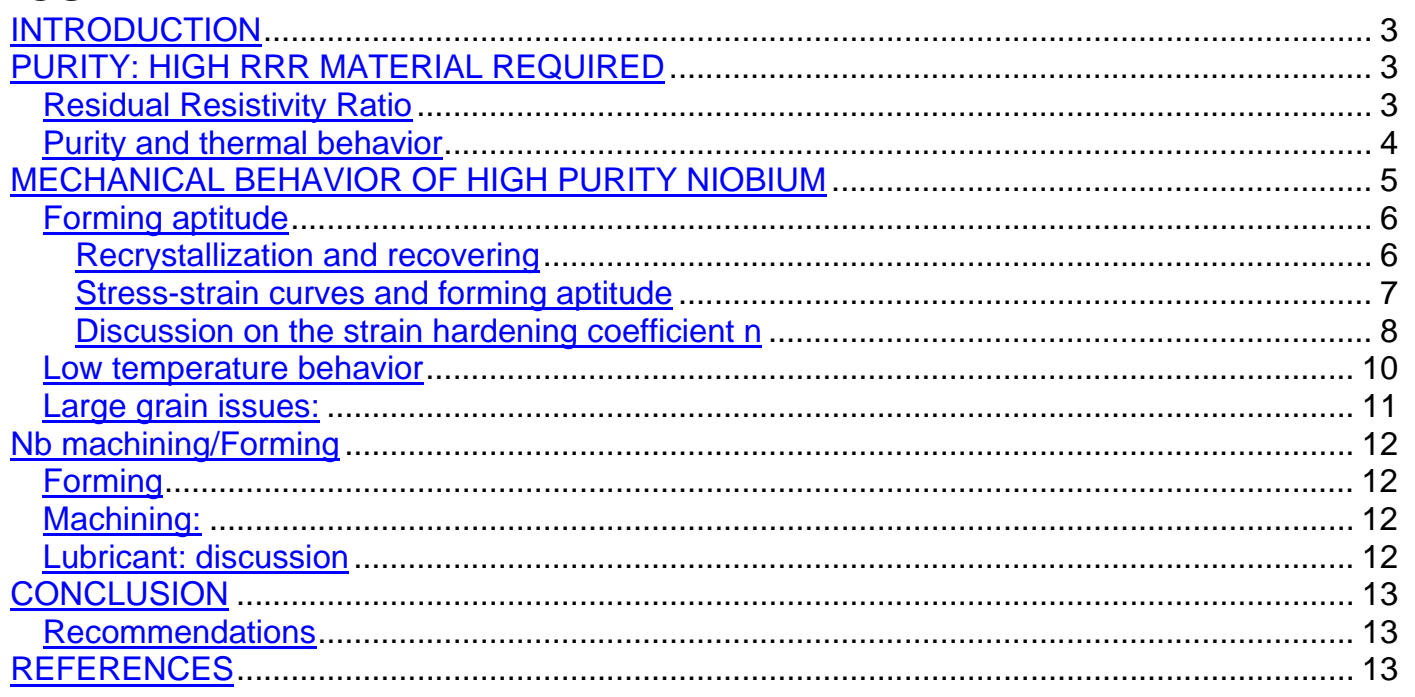

\section{INTRODUCTION}

It is important to distinguish among the properties of niobium, the ones that are related to the cavity's SRF performances, the formability of the material, and the mechanical behavior of the formed cavity. In general, the properties that dictate each of the above mentioned characteristics have a detrimental effect on one another and in order to preserve the superconducting properties without subduing the mechanical behavior, a balance has to be established. Depending on the applications, some parameters become less important and an understanding of the physical origin of the requirements might help in this optimization.

SRF applications require high purity niobium (high RRR), but pure niobium is very soft from fabrication viewpoint. Moreover conventional fabrication techniques tend to override the effects of any metallurgical process meant to strengthen it. As those treatments dramatically affect the forming of the material they should be avoided. These unfavorable mechanical properties have to be accounted for in the design of the cavities rather than in the material specification.

The aim of this paper is to review the significance of the important mechanical properties used to characterize niobium and to present the optimal range of values. Most of the following information deals with the specification of sheets for cell forming unless otherwise noted.

\section{PURITY: HIGH RRR MATERIAL REQUIRED}

\section{Residual Resistivity Ratio}

The purity of a metal can be characterized by its residual resistivity ratio (RRR), which is defined as the ratio of the electrical resistivity at $295 \mathrm{~K}$ to the resistivity at $0 \mathrm{~K}\left(\rho_{295 \mathrm{~K}} / \rho_{0 \mathrm{~K}}\right)$. The resistivity at a given temperature $\left(\rho_{T}\right)$ is proportional to the sum of resistivities from impurities $\rho_{\text {imp }}$, crystalline state (grain boundaries density, dislocations...) $\rho_{\text {cryst }}$, surface $\rho_{\text {surf }}$, and phonon interaction $\rho_{p h}(T)$, which is a function of temperature.

$$
\rho_{T} \sim \rho_{\text {imp }}+\rho_{\text {cryst }}+\rho_{\text {surf }}+\rho_{p h}(T)
$$

If the resistance measurements are performed on sufficiently large, well recrystallized samples, and at very low temperatures, then $\rho_{\text {surf }}, \rho_{\text {cryst }}$ and $\rho_{p h}(T)$ are negligible, and the residual resistivity depends mainly on the impurity content of the sample:

$$
\rho_{0 k} \approx \sum_{i} C i\left(\frac{\partial \rho}{\partial C}\right)_{i}
$$

In the case of niobium, the residual resistivity ratio has to be measured at its normal conducting state. For practical reasons it is more convenient to measure the resistance ratio, $R_{295 K} / R_{10 K}$ or $R_{295 K}$ 
$/ \mathrm{R}_{4.2 \mathrm{~K}}$. At $4.2 \mathrm{~K}$, induction of a magnetic transition from the superconducting state to the normal state is required.

The residual resistance $R_{0}$ can also be conveniently calculated from measurements conducted above the critical temperature $(\mathrm{Tc}=9.25 \mathrm{~K}$ for $\mathrm{Nb}$ ) and extrapolated to $\mathrm{T}=0 \mathrm{~K}$ using the simplified law

$$
R_{0}=R-\alpha R_{295} T^{3}
$$

Equation (2) is valid for many transition metals [1], and for niobium $\alpha$ is equal to $5.10^{-7} \mathrm{~K}^{-3}$ [2]. The measurement is done using the classical 4-wires method and can be handled in a simple liquid helium Dewar. Typical RRR values for various types of niobium are given in Figure 1.

\begin{tabular}{|c|c|c|c|c|c|c|}
\hline Origin & Commercial & RF application & $\begin{array}{c}\text { Post-purified } \\
\text { (cavities) }\end{array}$ & $\begin{array}{c}\text { Post-purified } \\
\text { (samples) }\end{array}$ & $\begin{array}{c}\text { Other } \\
\text { preparation }\end{array}$ & Theoritical \\
\hline RRR & $\mathbf{3 0 - 5 0}$ & $\mathbf{2 0 0 - 3 0 0}$ & $\mathbf{6 0 0 - 8 0 0}$ & Up to 1800 & $\mathbf{5 - 6 0 0 0}$ & $\mathbf{3 3 0 0 0}$ \\
\hline
\end{tabular}

Figure 1: typical RRR value for $\mathrm{Nb}$

The high RRR requirement is related to thermal conductivity rather than superconductivity. BCS theory predicts that the minimum BCS resistance corresponds to a RRR of around 30. Moreover, the surface RRR is expected to be somewhat different from the bulk RRR, and cavities with high as well as low RRR exhibit comparable $\mathrm{Q}_{0}$ at low field (see figure 2). Nevertheless their behavior at high field is fairly different and can be explained by their thermal properties.
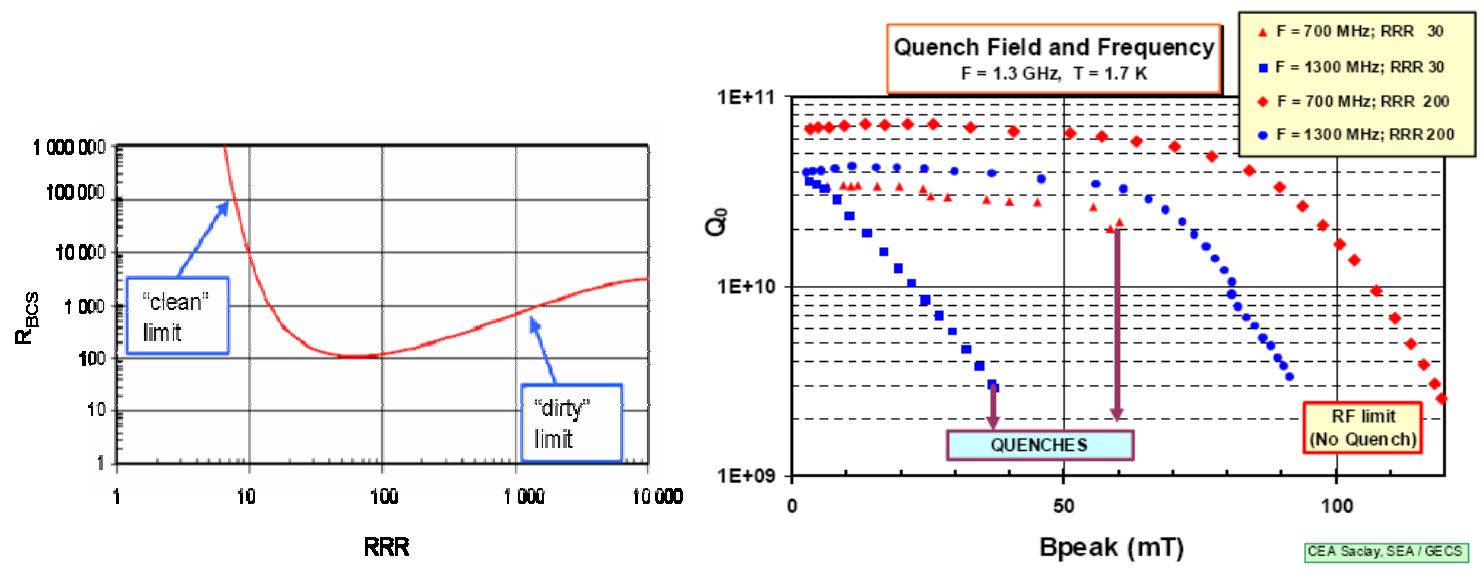

\section{Figure 2: BCS resistance of niobium at 4.2K, and examples of quench field of cavities for} different RRR and frequencies (Courtesy of $\mathrm{H}$. Safa).

Systematic improvement of the quench field is also observed after purification annealing of cavities with Titanium ${ }^{1}$. Purification annealing is seldom used these days as suppliers can provide materials with a RRR up to 400.

Good thermal conductivity helps to evacuate any thermal dissipation arising from the internal surface toward the external face of the niobium sheet which is in contact with helium, or to cool down parts that are outside the Helium vessel (cut-off tubes or couplers).

\section{Purity and thermal behavior}

In the $3 \mathrm{~K}-15 \mathrm{~K}$ range there is a direct relationship between RRR and thermal conductivity [4]. At $4.2 \mathrm{~K}$ the thermal conductivity is roughly equal to RRR/4 [5]. A superconductor is intrinsically a poor thermal conductor as a part of the (both electrical and thermal) conduction electrons are paired into Cooper pairs and thus cannot contribute to any heat transfer. To improve the thermal conductivity it is essential to get rid of the main scattering sources, i. e. interstitial light elements in the metal matrix. At lower temperature the major conduction mechanism is not related to electron but to phonon

\footnotetext{
${ }^{1}$ Ti reacts preferentially with Oxygen at temperatures around $800-1000^{\circ} \mathrm{C}$. The annealing cycle must include a sufficiently high temperature time in order to evaporate $\sim 1 \mu \mathrm{m}$ of Ti on the surface, and to allow diffusion of Oxygen to the surface. For details regarding the optimization of this process see e.g. [4].
} 
propagation. In such situations the scattering sources are rather crystalline defects and thus fully recrystallized samples exhibit a large phonon peak (see figure 3), even with a rather low RRR.

N.B.

- The phonon peak is a good indication of the crystalline state of the material. When a hot spot occurs, the surface can exhibit temperatures of several $\mathrm{K}$ compared to the $\sim 2 \mathrm{~K}$ of the remaining surface. It is the higher temperature contribution of thermal conductivity that matters.

- With the advent of new cryomodule designs, it is necessary to have parts that were typically made of low RRR material (cut-off tubes, couplers parts), to be made of very high RRR material, when no direct cooling is available. The low temperature conductance is the important parameter in this scenario.

- Another contribution to thermal transfer is the Kapitza resistance, that arises at the niobium-Helium interface. There is a lot of spreading in the possible values for Kapitza resistance of niobium, but as the efficiency of transfer from phonons to "rotons" ( equivalent of phonons inside a fluid) inside helium depends mostly on the effective surface area $S_{\text {eff, }}$ rough surfaces are expected to give rise to lower Kapitza resistance [6].

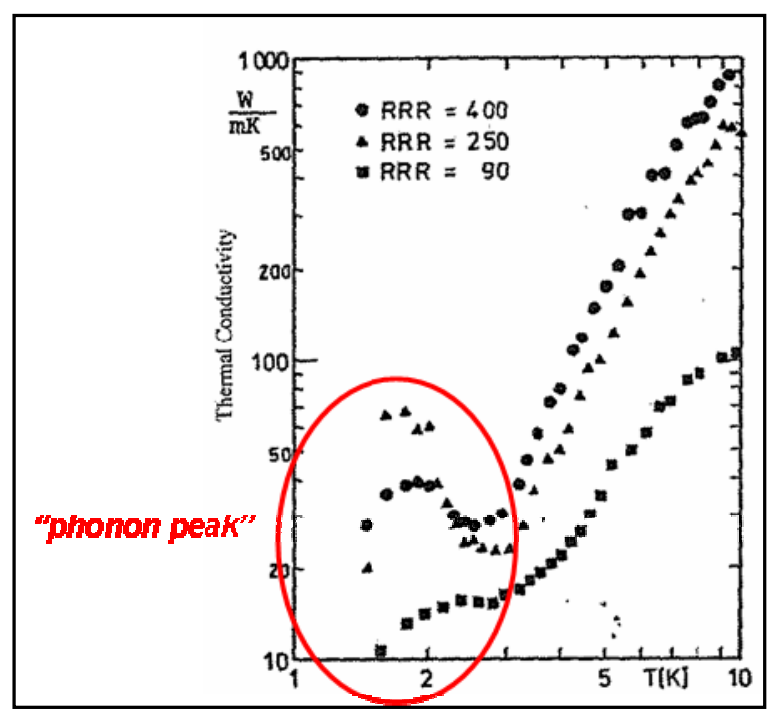

Figure 3: Thermal conductivity of various RRR samples

\section{MECHANICAL BEHAVIOR OF HIGH PURITY NIOBIUM}

The most commonly used mechanical properties are derived from tensile tests (stress-strain curves) and hardness measurements. Tensile tests describe the deformation behavior for an uniaxial case while in most forming processes bi-axial deformation occurs. In addition, specific mechanical tests are available and can be applied to complex forming processes such as hydroforming

In our case, two situations need observation:

- Small deformations: The material exhibits elastic behavior and deformations are reversible. Thus the parameters used to calculate mechanical resistance (stiffness) of finished objects are always linked to the elastic behavior of the material, i.e. Young's modulus (E), Poisson's ratio $(v)$, and to some extent Yield Strength or Elastic Limit $\left(\sigma_{0.2}\right)$. Tensile curves provide an estimation of the elastic properties of a material, but the values generally include measuring set-up error in the order of $5-10 \%$.

- Plastic deformation. Forming processes necessitate the overcoming of the elastic behavior of the material to obtain formability. The properties that dictate the plastic behavior depend on the material rheology and rupture information, and will be discussed in the next section.

Some of the mechanical properties change dramatically with the temperature, and/or thermomechanical history of the sample material. In particular, annealing (at $800^{\circ}$ or higher) of the cavities works as a "reset" and will erase the effects of cold working from the previous forming steps. Thus, the properties used to calculate the mechanical resistance of a finished cavity must be those of well annealed, fully recrystallized niobium. At a given temperature and purity these parameters have a fixed value and cannot be modified. We will see hereafter that they are not in favor of mechanical resistance.

The Young's modulus (E), and the Poisson's ratio (v), are intrinsic properties of a given material. Moreover, $v$ does not vary much from one metal to the other, and the "official value" for niobium is $v=0.397$ [7]. This parameter defines the lateral contraction of a specimen under a tensile load.

In the same way, $E$ is also an intrinsic property and the "official" value for niobium is $E=104.9$ GPa [7]. This modulus depends only on the lattice properties at a given temperature, and it should not 


\section{Antoine, M. Foley, N. Dhanaraj Last revised August 25, 2006 Physical properties of niobium and specification of superconducting cavities}

vary from one sample to another. Thus most of the discrepancies found in the literature can be attributed to variations in equipment and calibration. (One has to be aware that the measurement of such parameters is always delicate and requires sophisticated equipment). The Young's modulus can vary with crystalline orientation, which can be an issue in the case of single-crystal or large grain material, and it is expected to be slightly higher at cryogenic temperatures.

The Young's Modulus is a measure of the "stiffness" of a material during elastic deformation. The elastic modulus (E) for niobium is quite high, and it can be considered as a fairly "rigid" material as long as we stay under the elastic limit.

The last parameter used to predict the mechanical resistance is the elastic limit, or Yield Strength, and is generally obtained from a tensile test. This parameter can have values as low as 35$70 \mathrm{MPa}$ for well annealed niobium to some $100^{\text {eds }} \mathrm{MPa}$ for heavily deformed samples. Even for a fully recrystallized material, this parameter will depend strongly on the crystalline orientation and/or the texture, and on the previous "history" of the material; i.e. on the temperature and duration of the last annealing. As purification annealing occurs at fairly high temperature, the niobium cavities are actually made of very smooth material.

NB. In the past, a common misconception among the RF community has been that material with high yield strength would yield better strain resistant cavities. This is not true, as heat treatment (2 hours at $\sim 800{ }^{\circ} \mathrm{C}$ ) erases most of the cold working inside the material. One major drawback of this practice is that suppliers often deliver material with high Yield Strength values, but which has not been fully recrystallized. This can also be achieved by means of a "skin pass", which is a slight surface rolling process performed following the recrystallization annealing, and has the capability of artificially enhancing the yield strength of an incompletely recrystallized material.

Skin pass is widely used in the deep-drawing industry to improve the surface state and/or prevent "orange peeling" effect. It reduces the occurrence of large grains near the surface, which act as the origin of surface roughness. The drawback of employing skin pass is that it slightly hardens the material, and further annealing between this skin pass and a first forming step should be avoided.

In the case of very pure metals, this operation is always difficult to control, and is hardly reproducible. Moreover, this material is usually more difficult to form as it is "harder" than foreseen, and it is necessary to adapt the actual forming effort to each delivered batch, which jeopardizes the reproducibility of shapes.

\section{Formability}

Two parameters play a major role in the formability of metals: the grain size and the strain hardening coefficient, which can be calculated from the stress-strain curves (uniform plastic deformation). Small grain size is usually required in order to prevent the appearance of "orange peel". As material deformation depends on the crystalline orientation, in the case of a polycrystalline material some grains tend to deform less than the others, giving rise to a certain roughness termed as "orange peel". If the mean grain size is small, this effect is not very noticeable. Moreover, smaller grains accommodate a more uniform deformation. Small and uniform grain size is a strong requirement for good formability. The following two sections will address the means of achieving small grains and the other mechanical properties that influence the formability of $\mathrm{Nb}$.

\section{Recrystallization and recovering}

Recrystallization occurs upon heat treatment following a deformation step. If the deformation is over $65 \%$, uniform nucleation and small equiaxial grains ${ }^{2}$ can be obtained. Recrystallization temperature will decrease with increasing purity: it ranges from $900-1000^{\circ} \mathrm{C}$ for commercial $\mathrm{Nb}$ (RRR $\leq 100$ ) to $\sim 800^{\circ} \mathrm{C}$ for RRR $\sim 300$, and is expected to be around $750^{\circ} \mathrm{C}$ for RRR $>400$. Recovering mostly occurs at the same temperature as that of recrystallization, although it has been reported that in the case of a very slow annealing rate, it can start at lower temperatures. Recrystallization is recommended prior to any forming step as it helps remove the effects of cold working and achieves smaller grains. An added advantage to this heat treatment is that it removes hydrogen contamination that sometimes occurs during surface etching.

The deformation step prior to recrystallization is critical to achieving uniform grain size. Indeed, when recrystallization occurs, the final grain size depends on the initial amount of deformation, as shown in figure 4.

\footnotetext{
${ }^{2}$ Deformation and cold work introduce a lot of dislocations inside the material grains. These dislocations tend to reassemble and form "cells" inside the grain that will initiate the smaller grains nucleation. Equiaxiality of grains is the sign that recrystallization is completed.
} 
Physical properties of niobium and specification of superconducting cavities

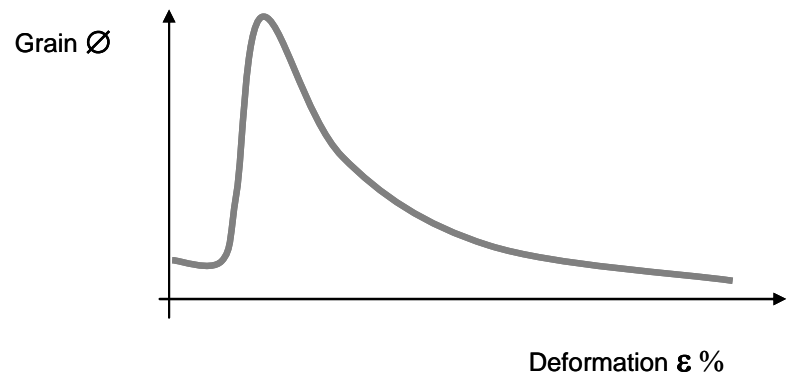

Figure 4: Grain diameter $\Phi$ after recrystallization as a function of initial stress ratio $\varepsilon$.

Materials subjected to smaller amounts of deformation recrystallize with very large grain diameters compared to heavily deformed ones. This post-recrystallization property is widely used to control grain size after a primary deformation (like rolling, cold extrusion, etc.). Discrepancies in grain size as showed in figure $5 \mathrm{a}$ often give rise to tearing during forming processes. Figure $5 \mathrm{~b}$ shows a well formed material despite large grain constitution. These types of grain distributions are directly linked to the rolling + recrystallization process.
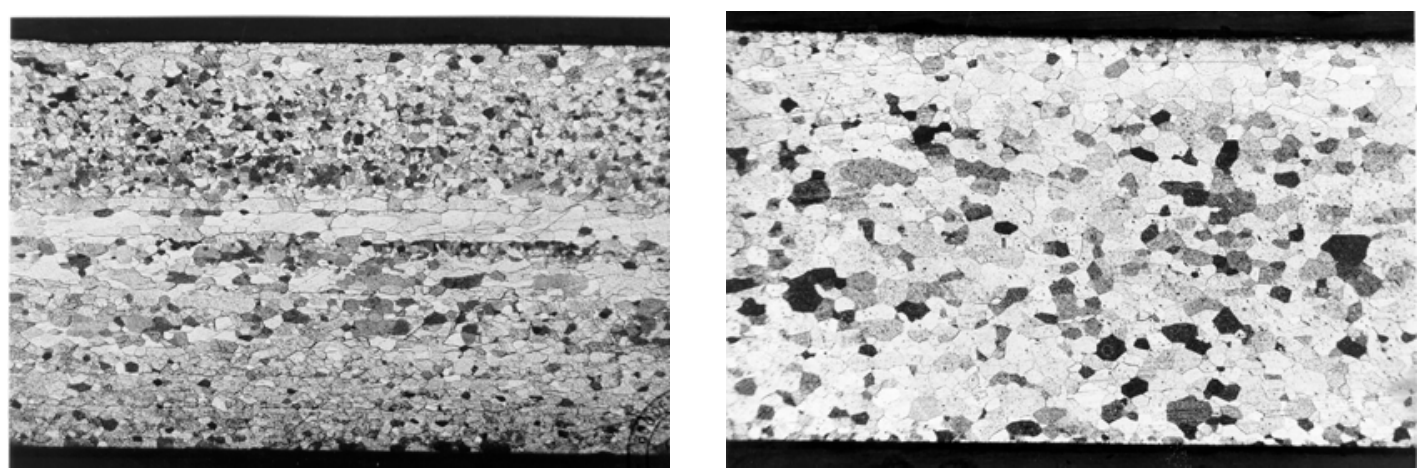

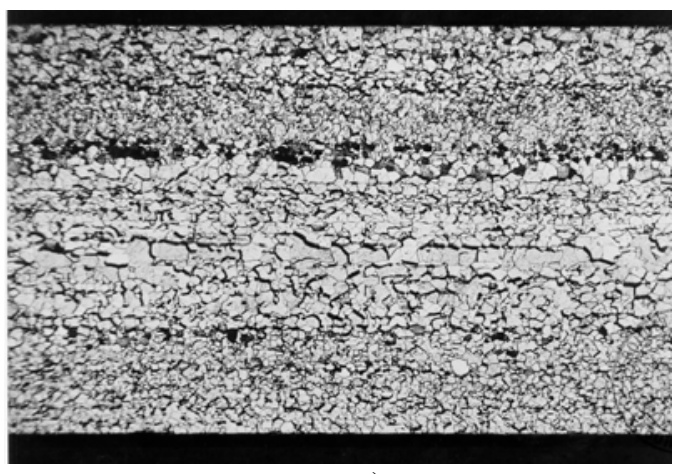

a)

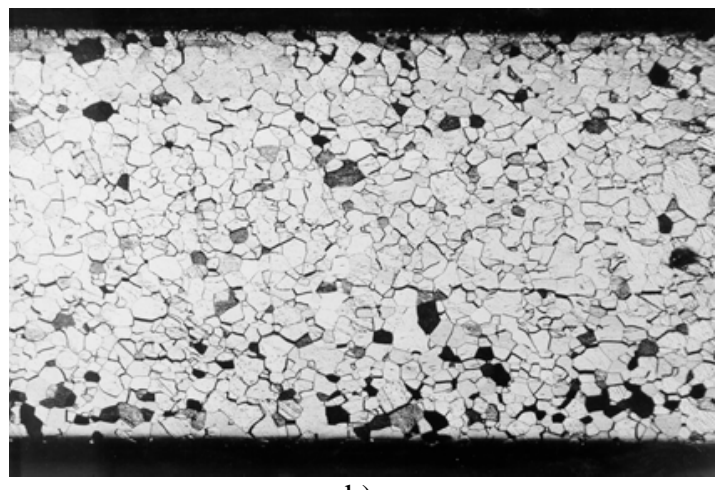

b)

Figure 5 : Macroscopic view of cuts on different niobium sheets (2 $\mathrm{mm}$ thick) : a)high level of tearing batch. b) normal forming batch. Note the grain boundary alignments in case a)

Stress-strain curves and formability

Often times some niobium batches exhibit "good" formability, while some pose major problems such as, intensive forming effort, high elastic recoil, anisotropy and even tearing while being formed. These problems can be correlated to the mechanical properties of niobium, which are directly linked to its crystalline state and not only the grain size.

Table 1 outlines the main mechanical properties of two batches of niobium from the same supplier, with RRR 300; batch A exhibits good formability, while batch B exhibits poor formability. Figure 6 shows the stress-strain curves of the two batches which graphically explain the observed behavior. 


\begin{tabular}{|c|c|c|}
\hline $\begin{array}{l}\text { Mechanical properties } \\
\text { a) IBatch }\end{array}$ & A & $B$ \\
\hline Yield Strength $\sigma_{0.2}(\mathrm{MPa})$ & 66 & 150 \\
\hline Tensile Strength $\sigma_{\mathrm{m}}(\mathrm{MPa})$ & 180 & 183 \\
\hline Elongation $\mathrm{A}(\%)^{\mathrm{b})}$ & 59 & 40 \\
\hline Strain Hardening Coef. $\mathrm{n}^{\mathrm{c}}$ & 0.31 & 0.10 \\
\hline Hardness Hv & 56 & 65 \\
\hline $\begin{array}{l}\text { Grain size (ASTM) } \\
\text { - core } \\
\text { - surface }\end{array}$ & $\begin{array}{l}6 \\
6\end{array}$ & $\begin{array}{l}5 \\
6\end{array}$ \\
\hline Formability & GOOD & BAD \\
\hline
\end{tabular}

TABLE 1
Notes:

a) The experimental measurements depend on eventual textures induced by deformation and can vary with the sample orientation. Figures presented here are mean values.

b) Total elongation; In the case of forming, the relevant parameter is uniform elongation (before necking)

c) $n$ is deduced from a tensile curve by curve fitting the plastically deformed portion with the formula $\sigma \alpha \varepsilon^{n}$. See discussion in the following section $\S$
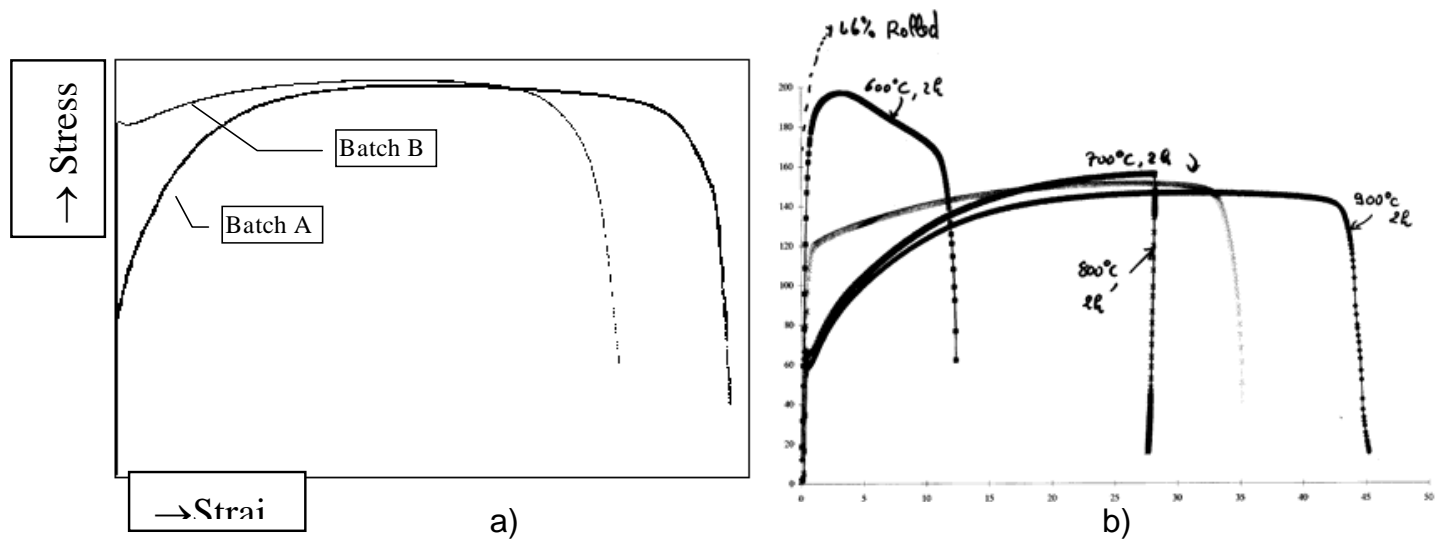

Figure 6: Stress-Strain Curves from Tensile Tests a) for two different niobium batches of the same purity range. Batch A: good forming behavior, Batch B: bad forming behavior. b) upon rolling and recrystallization for 2 hours at different temperatures.

The above example helps determine the properties required for good formability:

- A low tensile strength, which indicates better formability

- Enhanced elongation. (The figure of interest in the case of forming is the "uniform" elongation before necking and not the total elongation).

- A high strain hardening coefficient $\mathbf{n}$ (definition: see discussion below).

All these parameters vary significantly with the thermo-mechanical behavior of the material, especially, in the case of BCC metals like niobium. Only fully recrystallized material can exhibit such properties.

When rolling is not performed efficiently, i.e. if thickness reduction is not large enough, the surface will undergo a lot of deformation while the core of the material will be only slightly deformed. Upon recrystallization, this under-deformed region tends to have larger grains compared to the surface region, thus leading to the features observed in fig.5a). Thus it is very important to have a uniform grain distribution throughout the material and all grains should belong to the same ASTM category.

\section{Strain hardening coefficient $\mathrm{n}$}

Strain hardening coefficient is also a very important parameter and is a measure of the resistance of the material to necking. When a local deformation occurs, the necked region of the material is "hardened", and becomes more (as the value of $\mathrm{n}$ increases) resistant to further deformation. This results in the deformation of softer parts of the material away from the neck. The higher the $\mathrm{n}$, the more uniform the deformation and more delayed the appearance of localized necking. Qualitatively, $\mathbf{n}$ describes the "curvature" of the tensile curve; $\mathbf{n}$ can be high only if the difference between the Yield strength and the tensile strength is high. 
C. Antoine, M. Foley, N. Dhanaraj

Last revised August 25, 2006

Physical properties of niobium and specification of superconducting cavities

The strain hardening coefficient is of much interest to the "deep-drawing community". It has been proved in the case of bi-axial deformation, that $\varepsilon_{\max }$ varies as $4 / 11(2 n+1)(r e f$ [8]), i. e. that an increase of $n$ favors bi-axial deformation as it delays instability (necking). For more details regarding this calculation and its discussion, see [8]. Thus it helps to have a high $n$ value. Table 2 shows the $n$ values of different materials found in literature. Note that in the case of a material with good formability like copper, $\mathrm{n}$ ranges between 0.3 and 0.45 . The $\mathrm{n}$ values for niobium are in the same order as that of copper and thus have good formability too; also the $\mathrm{n}$ values are not influenced much by the presence of impurities like $\mathrm{O}$ (samples $(\mathrm{C}$ to (e) in Table 2). On the other hand, the $\mathrm{n}$ value seems to be highly influenced by the metallurgical state of the metal. 
Physical properties of Niobium and specifications for fabrication of superconducting cavities

\begin{tabular}{|l|l|l|l|}
\hline Metal & $\mathbf{n}($ ref [8]) & $\mathbf{N b}$ & $\mathbf{n}$ \\
\hline $\begin{array}{l}\text { Softened } \\
\text { steel }\end{array}$ & 0.15 & a) & $0.075-$ \\
0.25 & & 0.287 \\
\hline $\begin{array}{l}\text { austenitic } \\
\text { steel 18-10 }\end{array}$ & $0.4-0.5$ & b) & 0.45 \\
\hline aluminum & $0.07-027$ & c) & 0.45 \\
\hline copper & $0.3-0.47$ & d) & 0.45 \\
\hline zinc & 0.1 & e) & 0.45 \\
\hline nickel & 0.6 & f) & 0.10 \\
\hline & & g) & 0.31 \\
\hline
\end{tabular}

a) ref [8], the same $\mathrm{Nb}$ material, mechanical state ranging from heavily deformed to fully recrystallized

b) ref. [12], pure $\mathrm{Nb}$

c) ref. [12], pure $\mathrm{Nb}+80 \mathrm{Wppm} O$

d) ref. [12], pure $\mathrm{Nb}+230 \mathrm{Wppm} \mathrm{O}$;

e) ref. [12], pure $\mathrm{Nb}+330 \mathrm{Wppm} \mathrm{O}$; All samples in a well recrystallized state

f) Batch B: high failure rate when formed

g) Batch A: high success rate when formed Note: when not explicitly found in reference, $n$ is experimentally calculated with a graphic method described in ref [10] where $n$ is the slope of the straight part of the curve $\ln (s)$ $=f(\ln (e))$

\section{Low temperature behavior}

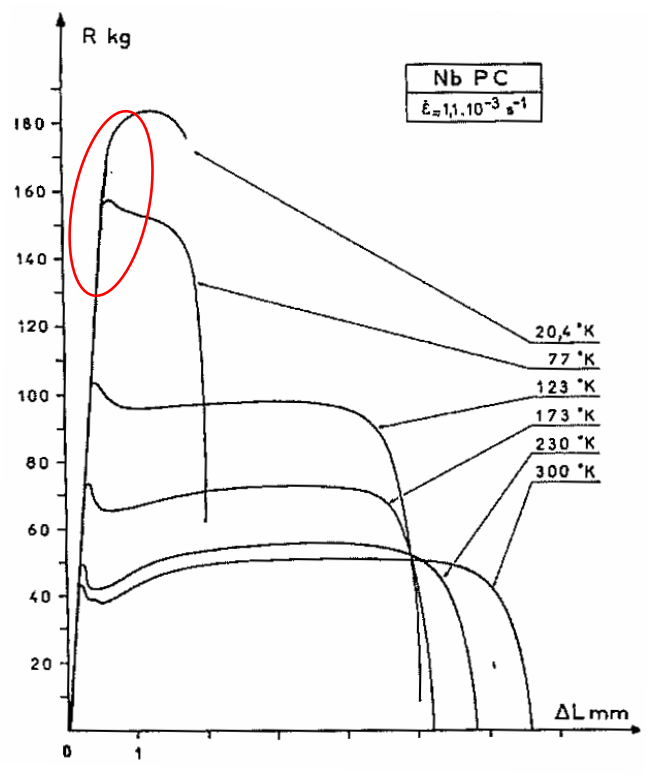

a)

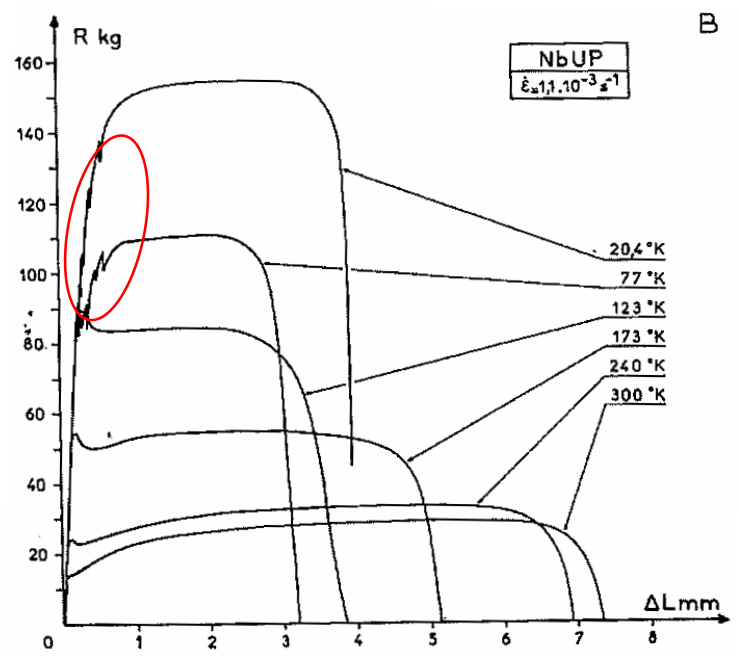

b)

Figure 7: stress-strain curves as a function of temperature for a) commercial and b) ultrapure niobium (from ref [10]). Red circle shows the onset of twinning (irreversible deformation)

Figure 7 shows the stress-strain curves for commercial (RRR 30) and ultrapure Nb (RRR300) for temperatures between $20 \mathrm{~K}$ and $300 \mathrm{~K}$. A decrease in the temperature reduces the ductility of the material while improving its yield strength and ultimate strength. The young's modulus is expected to be a little higher, but the $10-15 \%$ enhancement observed from stress-strain curves might arise from experimental set-up. At lower temperatures, the first steps of deformation occur by twinning (red circles on figure 7) and the material is still rather rigid, but the yield strength does not increase and stays constant. At $4.2 \mathrm{~K}$, fully recrystallized ultra pure niobium has a yield strength of $400 \mathrm{MPa}$ and ultimate strength of about $900 \mathrm{MPa}$ [12], but these values can vary significantly with purity or strain hardening, if present in the material. The material is brittle, i.e. no plastic deformation after twinning is observed. Figure 8 shows the variation of ultimate strength and yield strength (mean value $^{3}$ )

\footnotetext{
${ }^{3}$ Around and above room temperature, the stress-strain curve exhibits some times a lower Y.S. and an upper Y.S. This behavior is related to pinning of dislocation on interstitial atoms. They need to be given some activation energy to start to move.
} 
Physical properties of Niobium and specifications for fabrication of superconducting cavities C.Z. Antoine, M. Foley, N. Dhanaraj

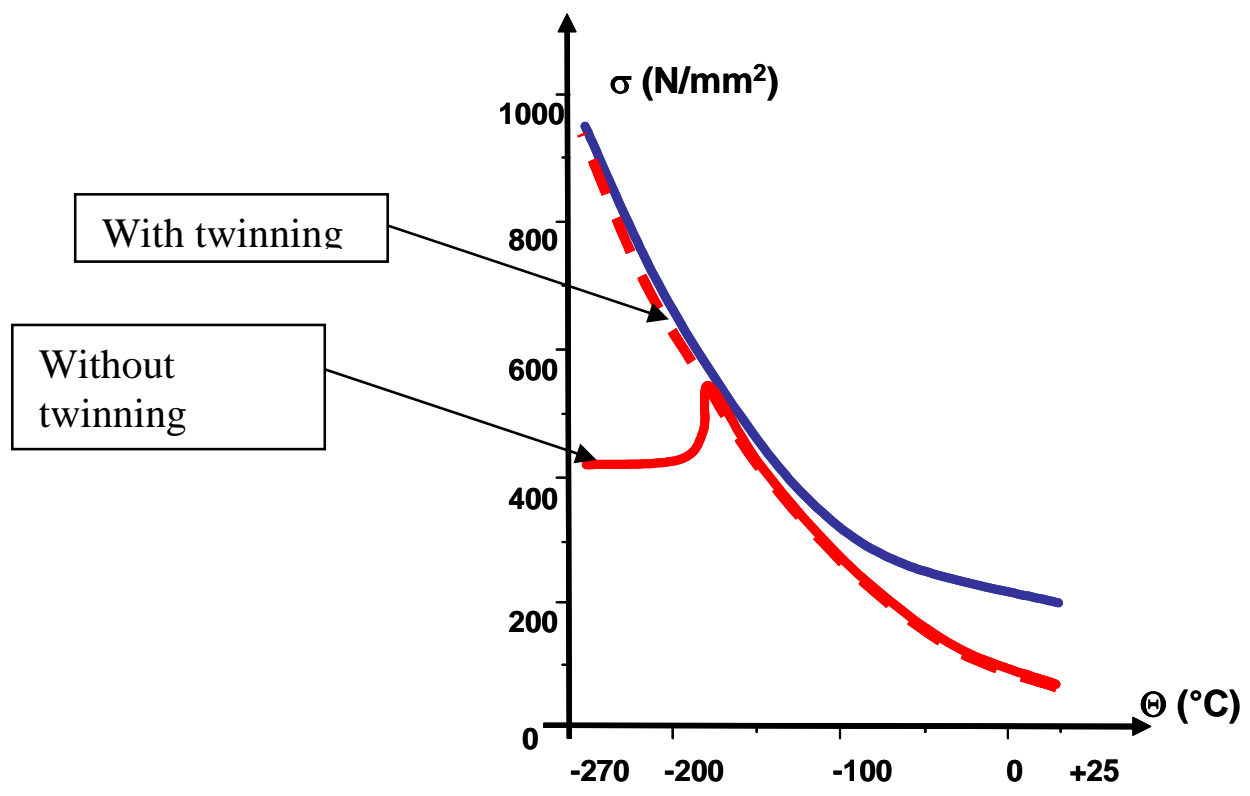

Figure 8: Behavior of ultrapure $\mathrm{Nb}$ (grain diameter $\sim 30 \mu \mathrm{m}$ ) with temperature[8]. Blue line: ultimate strength, red line: yield strength (dotted red: includes the apparition of twinning : the deformation is irreversible but keeps small). After [10].

\section{Large grain issues:}

The use of large grain or single crystal blanks directly sliced from the ingot was proposed recently by JLab [13]. This material exhibits similar properties as fine grain material from the SRF point of view and could cost less, but this aspect needs further study.

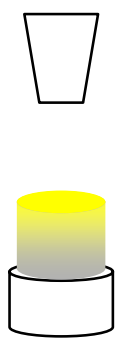

EB melting

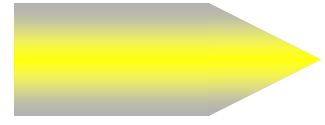

Hot forging (air)

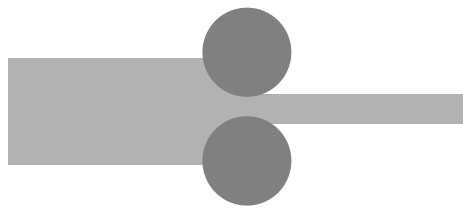

Cold rolling +
recovering

Figure 9: Fabrication scheme for fine grain Nb blanks

Figure 9 shows a schematic picture of the main steps involved in the fabrication of fine grain niobium. Several successive electron beam melts under vacuum help obtain a very high RRR material. As fine grain material is better from a forming perspective, it is necessary to heavily deform the starting material. This is done partially through hot forging, which also allows shaping the billet into a form that can be easily fed into the rolling machine, followed by several deformation/annealing steps. During the hot forging process, which is done in an open environment, the high RRR material from the billet can be heavily oxidized and the RRR decreases drastically.

By slicing directly from the initial billet one can save several steps of processing, conserve very high RRR, and also save on the cost.

The use of large grain or single crystal material has several drawbacks too. As nearly all the mechanical properties are orientation dependant, forming can result in a highly anisotropic material, and thus choosing a proper grain orientation is critical. Fortunately, very pure material is very ductile, and this should partially compensate for the undesirable effects during forming. In the case of large 
Physical properties of Niobium and specifications for fabrication of superconducting cavities C.Z. Antoine, M. Foley, N. Dhanaraj

grain material, all the grains will not deform uniformly under strain, and this could result in a phenomenon analogous to "orange peel", i.e. steps at the grain boundary. This can be overcome with appropriate mechanical polishing, for instance barrel polishing. Recrystallization at the welding seam is also another issue. It has been observed in the past that no recrystallization occurs when there is an equivalent orientation between two welded parts. It is also necessary to fully establish that half cells of the same orientation exhibit identical behavior on the whole periphery. If no recrystallization occurs, the surface of the cavity should be very smooth, even after a BCP (buffered chemical polishing), and this would eliminate the need for Electropolishing. Nevertheless, even with some recrystallization these cavities should have similar SRF properties as fine grain material.

\section{Nb machining/Forming}

As stated before, niobium is very soft and acts like soft copper or lead. Detailed information on the mechanical properties, machining techniques, etc can be found on the web. One website is given below:

http://www.wahchang.com/WahChang/pages/products/data/pdf/Niobium\%20Machining\%20\&\% 20Forming\%200perations.pdf

The information on the above website holds true only for Niobium alloys such as $\mathrm{NbTi}$, or commercial $\mathrm{Nb}$. High purity $\mathrm{Nb}$ is discussed below.

\section{Forming}

In the case of high pressure forming processes such as spinning or heavy deep drawing, niobium has a tendency to stick to the tools. Therefore special lubricants and die materials are required: brass, bronze; (Be-Cu or steel have also been used). In the case of forming processes involving smaller deformations, such as half cell deep drawing or hydroforming, aluminum tools are commonly used without the sticking problem.

\section{Machining:}

Niobium has a tendency to gall or to seize to tooling and this requires the use of tools with specific angles and proper lubrication. Tools must be very sharp and run at high speeds and steel tools work better than carbide tools. Manual feed (rate at operator's discretion) helps in rapid removal of niobium chips. While working with high RRR material, the speeds must be increased by a large factor compared to ones found in the literature, (cutting speed $\sim 250-270 \mathrm{rpm}$ for steel and $350-$ $400 \mathrm{rpm}$ for carbon tools, for details see [[14]).

\section{Lubricant: discussion}

The choice of a proper lubricant and its interaction with the niobium surface has been a major topic of discussion. Nevertheless one cannot avoid the effects of surface contamination or cold working upon any type of forming of niobium. Priority should be given in accordance to the ensuing cleaning procedure.

Therefore silicon type oil must be avoided, as it is very difficult to eliminate. Light hydrocarbon molecules that can be effectively rinsed/degreased are preferable (e.g. ethanol, water soluble hydrocarbon...).

As particle contamination is also an issue in SRF applications, lubricants with particle suspension like graphite or $\mathrm{MoS}_{2}$ should be generally avoided, although appropriate cleaning procedures help overcome this problem.

Any organic lubricant reacts with freshly cut $\mathrm{Nb}$ surfaces, but it results only in surface contamination that will be eliminated by subsequent chemical etching. 
Physical properties of Niobium and specifications for fabrication of superconducting cavities

\section{CONCLUSION}

\section{Recommendations}

- Need for high thermal conductivity:

-High RRR material

- Sheet material (forming)

-Recrystallized material $\left(800^{\circ} \mathrm{C}, 2 \mathrm{hrs}\right)$

-Uniform grains without different local grain size

-Small grain size (ASTM 5 or finer)

--Yield strength should be $50<\sigma 0.2<80 \mathrm{Mpa}$

With such conditions, we can achieve a fairly high strain hardening coefficient $(n>0.3)$, and a more uniform distribution of deformation in the material. These conditions do not affect purity and RF properties, but are very helpful to any forming process.

- Rod (drilling, machining)

-Lubricant must be easily cleaned (no SILICONE grease !!!)

-Recrystallization is not mandatory prior to working (unless the material has already been subjected to extensive cold working)

-Recrystallization/recovering must be done after machining (and proper cleaning) in

order to get rid of cold work and possible hydrogen contaminations.

-Small grain material $=$ better (because of etching )

For low RRR material, it is also worthwhile to have a well recrystallized material. Meanwhile the hardness, tensile strength and yield strength will be a little higher than for high RRR. Typical values for material with RRR of 30 are: $\sigma_{0.2} \sim 230-250, \sigma_{\max } \sim 280-300, \mathrm{Hv} \sim 60-70$. This also means forming is more difficult compared to high RRR material.

\section{REFERENCES}

1. Meaden, G. T., "Electrical resistance of metals", London: Heywood books, 1965.

2. Safa, H. et al., "Specific Resistance Measurement of a Single Grain Boundary in Pure Niobium", 9th Workshop on RF Superconductivity-1999, edited, Santa Fe, NM, USA.

3. Safa, $\mathrm{H}$. et al., "Advances in the purification of niobium by solid state gettering with titanium", Journal of alloys and compounds 232, 281-288 (1996).

4. Koechlin, F. and Bonin, B., "Parametrisation of the niobium thermal conductivity in the superconducting state", Superconductor Science and Technology 9, 453-460 (1996).

5. Padamsee, H, Knobloch, J., Hays,T., "RF superconductivity for accelerators", Wiley series in beam physics and accelerator technology, Wiley \& sons Ed., (1998)

6. . J. Amrit, C.Z. Antoine, M. X. Francois, H. Safa., "On intrinsic thermal limitations of superconducting cavities: Kapitza resistance", Advances in Cryogenic Engineering, 2002. 47(A): p. 499-506

7. J. Emsley, "The Elements", Oxford University Press, Oxford, UK, 3rd edition, 1997.

8. E Massoni "'Approche scientifique des procedés de mise en forme-Vol 3 : Emboutissage (Scientific approach to forming processes- Vol 3 : deep drawing), courses from the "Ecole des mines de Paris "(1994).

9. C.Z. Antoine, D. Cochet, "Hydroforming of niobium Rf cavities", 7th workshop on RF superconductivity, Oct. 17-20, 1995.

10. P mazot, PhD Thesis, Poitier, (1970)

11. JF Fries, PhD Thesis, Orsay, (1972)

12. Saclay, unpublished results.

13. http://www.jlab.org/news/releases/2005/crystal.html

14. Fermilab technical note TD- TD-06-049: PARAMETERS FOR MACHINING NIOBIUM AT FNAL, C. Antoine, D. Snee, J. Mueller, D. Burk, M. Foley. To be published. 